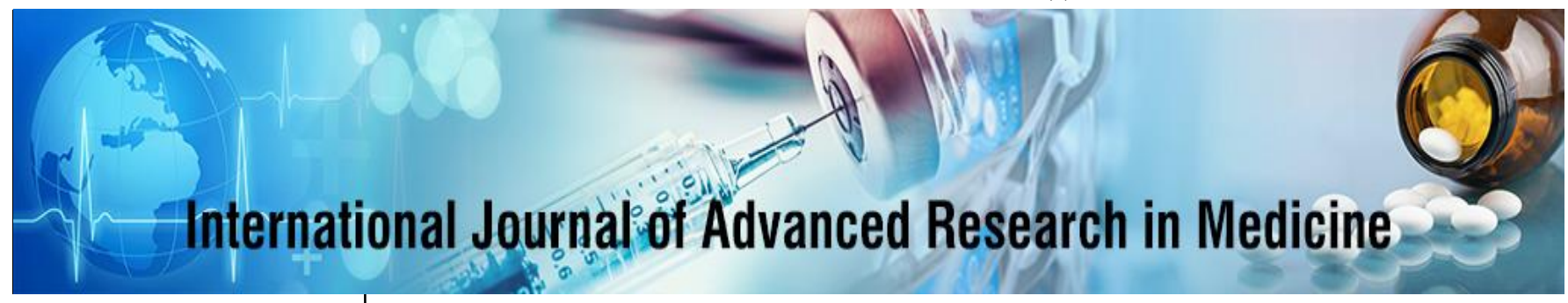

E-ISSN: 2706-9575

P-ISSN: 2706-9567

www.medicinepaper.net

IJARM 2021; 3(2): 220-223

Received: 13-08-2021

Accepted: 20-09-2021

Dr. Sahib Singh Kailday Medical Officer, Dhawan Hospital, Ludhiana, Punjab, India

Dr. Aman Mahey

Medical Officer, Dhawan

Hospital, Ludhiana, Punjab, India
Corresponding Author: Dr. Sahib Singh Kailday Medical Officer, Dhawan Hospital, Ludhiana, Punjab, India

\section{A hospital based research to determine the incidence and severity of vitamin d insufficiency in people with type 2 diabetes}

\author{
Dr. Sahib Singh Kailday and Dr. Aman Mahey
}

DOI: $\underline{\text { https://doi.org/10.22271/27069567.2021.v3.i2d.249 }}$

\begin{abstract}
Aims: The aim of the study to assess the prevalence and severity of vitamin D deficiency in type 2 DM.

Material and methods: A 5 months case-control study was carried out at Dhawan Hospital in Ludhiana, Punjab, India, at the request of the researchers. A total of 240 volunteers participated in the study, with 120 type 2 diabetes patients serving as case participants (Group A) and 120 healthy volunteers serving as control participants (Group B). Laboratory testing on a regular basis CBC, FBS, RBS, PP2BS, haemoglobin A1C, blood urea, serum creatinine, lipid profile, urine albumin, and Vitamin D3 levels were all measured using conventional techniques at the institute's central laboratory, according to the guidelines.

Results: When comparing groups A and B, the mean age of group A (case) was 51.429 .25 years, while the mean age of group B (control) was 50.1210 .18 years. The prevalence of low vitamin D levels in the healthy population was just 19.2 percent, while the prevalence in the diabetic population was 84.2 percent. Vitamin D levels were found to be sufficient, insufficient, and deficient in 18.51 percent, 61.11 percent, and 16.67 percent, respectively, in patients with controlled diabetes according to $\mathrm{HbA} 1 \mathrm{C}$ criteria, but only 10.60 percent, 68.18 percent, and 24.24 percent in patients with uncontrolled diabetes, according to the same criteria. In contrast to diabetic patients with managed condition, a higher proportion of diabetic patients with uncontrolled state ( 24.24 percent) had overt vitamin D insufficiency (16.67 percent). There is a statistically significant relationship between the maintenance of euglycemia and the degree of Vitamin D deficiency in diabetic patients, as shown by a $\mathrm{p}$ value less than 0.05 .

Conclusion: All patients with type-2 diabetes should have their vitamin D levels checked, and those who are found to have insufficiency or shortage of vitamin D should be prescribed vitamin D supplements. In order to avoid vitamin D insufficiency, it is also necessary to maintain tight control over one's diabetic condition.
\end{abstract}

Keywords: Serum vitamin D level, Type 2 diabetes mellitus, Vitamin D deficiency

\section{Introduction}

Type-2 diabetes, is a chronic metabolic disease whose incidence is rapidly rising across the globe. Approximately 562 million people are expected to develop diabetes in the world by 2030, according to current estimates ${ }^{[1]}$. Despite the fact that the number of individuals with type 2 diabetes is growing in every nation, the majority of those affected live in developing countries, where the disease is quickly becoming an epidemic. Because of the growing worldwide burden of type 2 diabetes, researchers are re-examining the biology of this illness with fresh vigour. Insulin resistance and beta-cell failure are the two most important pathophysiologic abnormalities in type 2 diabetes. It is mainly caused by a complex interaction of genetic and environmental influences. The prevalence of type 2 diabetes varies from one geographical area to another as a result of variations in lifestyle and associated risk factors. In addition to traditional environmental risk factors such as obesity, physical inactivity, consumption of high-calorie foods, and stress, the involvement of specific dietary variables in the pathogenesis of type 2 diabetes is a notion that is still in its early stages. A growing body of data from many cross-sectional studies indicates that vitamin D plays an essential role in the regulation of blood glucose levels and that a vitamin D deficit may lead to the development of type 2 diabetes. Because it is now well-established that the active form of vitamin $\mathrm{D}$ is a hormone, it is no longer referred to as a vitamin. 
Instead, it is referred to as a hormone because it is involved in a wide range of physiological processes other than bone metabolism. Several mechanisms have been proposed to explain the positive effect of vitamin D on insulin secretion and sensitivity, including its direct effect on insulin secretion and sensitivity via activation of the vitamin D receptor on pancreatic beta-cells and insulin sensitive organs and its indirect effect on insulin secretion and sensitivity via regulation of calcium homeostasis ${ }^{[2,3]}$. In the biggest epidemiological research to date, drawn from the NHANES population, a dose-dependent inverse association between vitamin $\mathrm{D}$ and type 2 diabetes was discovered, with the lowest quartiles of vitamin $\mathrm{D}$ being associated with the greatest proportion of metabolic syndrome patients ${ }^{[4]}$. Exposure to sunshine, a healthy diet, and dietary supplements are the primary sources of vitamin D in the human body. 25-hydroxyvitamin D [25 $(\mathrm{OH}) \mathrm{D}]$ is formed in the liver as a result of the absorption of vitamin $\mathrm{D}$ from the skin and food. This substance is used to assess the vitamin D status of the patient. Although there is no universal agreement on what constitutes a normal level of vitamin $\mathrm{D}$, most experts agree that less than $20 \mathrm{ng} / \mathrm{ml}$ is indicative of vitamin $D$ insufficiency. Insufficiency in vitamin $D$ is indicated by a level between 20 and $29 \mathrm{ng} / \mathrm{ml}$, with a level higher than 30ng/ml being deemed adequate in vitamin $\mathrm{D}^{[5}$, 6]. Vitamin D deficiency or insufficiency is estimated to affect about 1 billion individuals globally, according to this definition. Even in the sunniest nations, such as India, vitamin D insufficiency is very prevalent, owing to the fact that the majority of the body's surface is protected from sunlight. Both type 2 diabetes and hypovitaminosis D are common in India, which is a developing nation. However, there is just a little amount of data accessible to observe the relationship between the two. The study's primary goal was to determine the prevalence and severity of vitamin D insufficiency in people with type 2 diabetes, as well as to document the impact of hyperglycemia on blood vitamin D levels.

\section{Material and Methods}

A 5 months case-control study was carried out at Dhawan Hospital in Ludhiana, Punjab, India, at the request of the researchers. Following the receipt of informed permission, a comprehensive history was obtained from the patient or his or her family.

\section{Methodology}

All patients were informed about the operation, including the method, risks, advantages, outcomes, and problems that may arise as a consequence of the treatment. A total of 240 volunteers were included in the research, 120 type 2 diabetic patients were included as case (Group A) and 120 healthy patients as control (Group B). Healthy people who are age and sex matched to the participants serve as controls. Patients with chronic renal disease, patients who had used calcium or vitamin D supplements within the previous three months, and patients who had a known chronic sickness were all precluded from participating in this investigation. Laboratory testing on a regular basis CBC, FBS, RBS, PP2BS, haemoglobin A1C, blood urea, serum creatinine, lipid profile, urine albumin, and Vitamin D3 levels were all measured using conventional techniques at the institute's central laboratory, according to the guidelines. It was determined that the value of blood vitamin D level was adequate if it was between 30 and $100 \mathrm{ng} / \mathrm{ml}$, insufficient if it was between 20 and $29 \mathrm{ng} / \mathrm{ml}$, and deficient if it was less than $20 \mathrm{ng} / \mathrm{ml}$.

\section{Statistical analysis}

The recorded data was compiled and entered in a spreadsheet computer program (Microsoft Excel 2010) and then exported to data editor page of SPSS version 19 (SPSS Inc., Chicago, Illinois, USA). Descriptive statistics included computation of percentages and means. Test applied for the analysis was chi-square test.

\section{Results}

In the study population, the mean age of group A (case) was $51.42 \pm 9.25$ years while that of group B (control) was $50.12 \pm 10.18$ years. Total 140 males and 100 females were enrolled in study. $65 \%$ of group A were male and 35 female. Group B (control) had $60 \%$ males and 40 female. On evaluation of investigation profile of both group A and group B, mean values of haematological parameters in form of haemoglobin, total count and platelet were within normal limit and comparable in both groups. Surprisingly mean value of renal function test parameters, blood urea and serum creatinine were within normal range for diabetic group also, though $10.5 \%$ of patients had abnormal serum creatinine value and it range from 2.22 to $4.2 \mathrm{mg} / \mathrm{dl}$. Mean value of all lipid profile component were in normal limit in both the group, but $35 \%$ of diabetic patients had dyslipidaemia and commonest dyslipidaemia was hypertriglyseridemia in 30\% patients. Frequency Distribution of Participants according to Severity of Vitamin D level noted. Prevalence of low vitamin D level in healthy population was only $19.2 \%$, while prevalence was $84.2 \%$ in diabetic group. (Table 1.)

Table 1: Severity grading of vitamin D deficiency in cases and controls

\begin{tabular}{|c|c|c|c|c|}
\hline & Case =120 & \% & Control=120 & \% \\
\hline Deficiency & 24 & 20.0 & nil & - \\
\hline Insufficient & 77 & 64.2 & 23 & 19.2 \\
\hline Sufficient & 19 & 15.8 & 97 & 80.8 \\
\hline
\end{tabular}

Table 2: The association of severity of vitamin D level with the category of diabetes control

\begin{tabular}{|c|c|c|c|c|}
\hline \multirow{2}{*}{ Diabetes control } & \multicolumn{3}{|c|}{ Vitamin D Level } & \multirow{2}{*}{ p value } \\
\cline { 2 - 4 } & Sufficient & Insufficient & Deficiency & \\
\hline $\begin{array}{c}\text { Controlled } \\
\text { Diabetic } \\
(\mathrm{N}=54)\end{array}$ & $10(18.51 \%)$ & $33(61.11 \%)$ & $9(16.67 \%)$ & $\leq 0.05(\mathrm{NS})$ \\
\hline $\begin{array}{c}\text { Uncontrolled Diabetic } \\
(\mathrm{N}=66)\end{array}$ & $7(10.60 \%)$ & $45(68.18 \%)$ & $16(24.24 \%)$ & 0.013 (Sig.) \\
\hline
\end{tabular}


In patients with controlled diabetes as per $\mathrm{HbA} 1 \mathrm{C}$ criteria, the prevalence of sufficient, Insufficient and Deficient Vitamin D was $18.51 \%, 61.11 \%$ and $16.67 \%$ respectively, where in patients with uncontrolled diabetes it was $10.60 \%$, $68.18 \%$ and $24.24 \%$ respectively. More number of diabetic patients with uncontrolled status $(24.24 \%)$ was having overt vitamin $\mathrm{D}$ deficiency in comparison to controlled status $(16.67 \%)$. There is a significant association between the maintenance of euglycemia and severity of Vitamin D level in diabetic patients, as the p value is less than 0.05 (Table 2). Pearson correlation test showed negative correlation between HbA1C level and mean vitamin D level in Diabetic group as $r=-0.266$, $p$ value $=<0.001$. It suggests as HbA1c level increase, the level of vitamin $\mathrm{D}$ decreases, so more severe the hyperglycaemia and poorer the control of diabetes status, there was more severe the vitamin D deficiency.

We also compare the mean value of vitamin D deficiency with the duration of Diabetes, but there was no significant relation between duration of diabetes and serum vitamin $\mathrm{D}$ deficiency ( $\mathrm{P}$ value $>0.5$ ).

Diabetic nephropathy was the most common micro vascular complication seen in type 2 diabetic patients. it was found that all three important microvascular complications: diabetic retinopathy, diabetic nephropathy and peripheral neuropathy did not have any significant correlation with serum Vitamin D level as $\mathrm{p}$ value is greater than 0.05 for all three parameters (Table 3).

Table 3: Comparison of mean vitamin D level with duration and micro vascular complication of diabetes mellitus

\begin{tabular}{|c|c|c|c|c|}
\hline & Parameters & & Mean value of vitamin D (ng/dl) & p value \\
\hline \multirow{4}{*}{ Duration of diabetes } & $0-5$ years & & $25.77 \pm 6.85$ & $\leq 0.05$ (NS) \\
\cline { 2 - 4 } & $5-10$ years & & $27.00 \pm 5.85$ & \\
\cline { 2 - 4 } & $>10$ years & & $26.12 \pm 3.82$ & $\leq 0.05$ (NS) \\
\hline Micro-vascular complication & Diabetic & Present & $24.11 \pm 5.02$ & \\
\cline { 2 - 4 } & Retinopathy & Absent & $26.31 \pm 5.22$ & \\
\cline { 2 - 4 } & Diabetic & Present & $27.11 \pm 5.45$ & \\
\cline { 2 - 4 } & Nephropathy & Absent & $25.41 \pm 6.35$ & \\
& Peripheral & Present & $24.24 \pm 4.69$ & \\
\hline & Neuropathy & Absent & $27.25 \pm 5.88$ & \\
\hline
\end{tabular}

Hypertension was most common co-morbidity found in diabetic group (18.33\%) followed by Ischemic Heart Disease $(5.83 \%)$. Serum vitamin D level was measured in all participants. 85.83 of diabetic population was having less than normal vitamin D level, while only $23.33 \%$ had less than normal vitamin $\mathrm{D}$ level in group $\mathrm{B}$ (control). Mean value of vitamin $\mathrm{D}$ in type 2 Diabetic patients was $26.33 \pm 6.94 \mathrm{ng} / \mathrm{dl}$ and mean value of vitamin $\mathrm{D}$ in healthy individuals was $35.67 \pm 4.88 \mathrm{ng} / \mathrm{dl}$. (Table 4 )

Table 4: Subgroup analysis- mean vitamin D level in diabetes patients in relation with age, gender and associated co-morbidities

\begin{tabular}{|c|c|c|c|}
\hline \multicolumn{2}{|c|}{$\begin{array}{c}\text { Parameters (Number of } \\
\text { patients) }\end{array}$} & $\begin{array}{c}\text { Mean value of } \\
\text { vitamin D }\end{array}$ & $p$ value \\
\hline \multirow{2}{*}{ Age group } & Below40 (91) & $25.32 \pm 5.75$ & \multirow{2}{*}{$\begin{array}{l}\leq 0.05 \\
(\mathrm{NS}) \\
\end{array}$} \\
\hline & $>40(29)$ & $25.02 \pm 3.19$ & \\
\hline \multirow{2}{*}{ Gender } & Male (78) & $28.65 \pm 5.86$ & \multirow{2}{*}{$\begin{array}{l}0.031 \\
\text { (Sig.) }\end{array}$} \\
\hline & Female (42) & $25.09 \pm 4.68$ & \\
\hline \multirow{2}{*}{ HTN } & Yes (22) & $21.66 \pm 4.22$ & \multirow{2}{*}{$\begin{array}{l}0.023 \\
\text { (Sig.) }\end{array}$} \\
\hline & No (98) & $25.68 \pm 6.81$ & \\
\hline \multirow{2}{*}{ IHD } & Yes (7) & $28.23 \pm 4.72$ & \multirow{2}{*}{$\begin{array}{l}\leq 0.05 \\
(\mathrm{NS})\end{array}$} \\
\hline & No (113) & $26.17 \pm 5.82$ & \\
\hline
\end{tabular}

Test applied: t-test

\section{Discussion}

Vitamin D insufficiency is a significant public health concern across the globe. A research conducted by Pfotrnhauer KM and colleagues found that the total global incidence of Vitamin D insufficiency is about 15 percent ${ }^{[7]}$. In India, on the other hand, the incidence of vitamin D insufficiency is between 50 and 90 percent in the normal healthy population ${ }^{[8]}$. In our research, the prevalence of vitamin D insufficiency in the general population was found comparable to the global prevalence but much lower than the prevalence seen in Indian studies. This low incidence in a healthy group was in direct conflict with the findings of previous Indian research. The results of another research on vitamin D levels, which was conducted in a comparable area, revealed that 16 percent of the healthy population had vitamin D insufficiency. It is known that vitamin D has an impact on calcium metabolism and, as a result, has an effect on the skeletal system; however, it also has extraskeletal effects on the cardiovascular system, endocrine diseases, and autoimmune illnesses, among other things. Several studies have shown that vitamin D has an important role in the functional control of the endocrine pancreas, especially the beta-cells, in humans.

India has previously been designated as the "Capital of Diabetes." Diabetes mellitus is recognised as a significant growing pandemic in India, with 41 million diabetes patients presently on the population rolls, with that number expected to rise to 70 million by the year 2025 . The fact that vitamin $\mathrm{D}$ has been shown to have an impact on the pathophysiology of diabetes, as well as the fact that vitamin D insufficiency is very common, led us to conduct this research in order to determine the effect of these two high prevalence illnesses on one another.

In a variety of studies conducted in various geographical regions and cultural backgrounds, researchers discovered a wide range of prevalence of vitamin D insufficiency in the diabetic population, with estimates ranging from $67 \%$ to $98 \%{ }^{[8-11]}$. We, along with Bashir et al. and IfigeniaKostoglou A et al. investigations, found a greater incidence of vitamin D insufficiency in diabetic patients when compared to healthy people; however, two other studies found no difference in prevalence between diabetic and healthy individuals ${ }^{[8-11]}$. We thus compared mean blood vitamin $\mathrm{D}$ levels in diabetes patients with those in a healthy population from a variety of studies. Several research, including ours, found that diabetes individuals had lower mean levels of vitamin $\mathrm{D}$ than the general population when compared to the general population ${ }^{[8,11,12]}$.

In our research, the mean vitamin $D$ level was shown to be lower in patients with uncontrolled diabetes than in patients with managed diabetes, compared to patients with 
controlled diabetes. Mukherjee B et al. demonstrated findings that were similar to ours. When comparing uncontrolled diabetes patients $(19.47 \pm 4.76)$ to managed diabetic patients $(23.63 \pm 3.71)$, the mean level of vitamin D is lower in the uncontrolled diabetic patients. According to the findings of Modi KD et al, vitamin D levels in patients with managed diabetes were $22.41 \pm 8.6$, while they were $19.91 \pm 8.3$ in patients with uncontrolled diabetes, a difference that was statistically significant ${ }^{[13]}$. Overall, insufficiency is more common than deficiency state in diabetic patients regardless of diabetic control status, but severe vitamin D deficiency is more common in patients who have uncontrolled diabetes than in patients who have controlled diabetes, according to the American Diabetes Association (24.24 percent and 16.67 percent respectively). A negative connection between HbA1C levels and blood vitamin D levels was found in the research, according to Pearson correlation analysis. It suggests that when the amount of haemoglobin A1C rises, the level of blood vitamin $\mathrm{D}$ decreases as well. It has also been discovered by Ifigenia-Kostoglou A et al. that 25(OH) D3 levels were negatively related with $\mathrm{HbA1c}$ levels when the patient and control groups were examined combined $(\mathrm{p}=0.008$ and $\mathrm{r} 2$ $=0.058$, respectively, in a linear regression study) ${ }^{[11]}$. According to a study conducted by Mukherjee B et al, there is a significant negative connection between Vitamin D levels and diabetes $(r=-0.94$ and -0.97$)$, with poorly managed diabetics having even lower Vitamin D levels ${ }^{[12]}$. Researchers Akshaykumar SV and colleagues found that vitamin D levels were negatively associated with $\mathrm{HbA1C}$ levels, but that the relationship was not statistically significant $(r=0.017, p \text { value } 0.741)^{[10]}$. The findings of this research, which showed an adverse association between vitamin D levels and glycaemic control, support the hypothesis that vitamin D plays a role in the development of type 2 diabetes mellitus.

The duration of diabetes and the existence of microvascular complications had no impact on the amount of vitamin D in the blood. We found no evidence of a connection between increasing age and vitamin D status in diabetes patients, and we were unable to locate any evidence of such an association in other research. It was shown that female diabetes patients have lower vitamin $\mathrm{D}$ levels than their male counterparts; the explanation for this may be due to decreased exposure to sunlight as a result of household activities. Among diabetic patients in our research, hypertension was the most frequent comorbidity (found in 18.33 percent of those who had diabetes). The findings of a study conducted by Shalini $\mathrm{P}$ et al. revealed that vitamin D insufficiency is more common in hypertension patients (80.4 percent) than in healthy people (67.7 percent) ${ }^{[14]}$. When compared to non-hypertensive diabetes patients in my research, hypertensive diabetic patients had lower vitamin D levels, which was statistically significant. Another comorbidity associated with diabetes was ischemic heart disease, although no statistically significant difference in mean Vitamin D levels was observed between diabetic individuals with and without ischemic heart disease.

\section{Conclusion}

All patients with type-2 diabetes should have their vitamin D levels checked, and those who are found to have insufficiency or shortage of vitamin D should be prescribed vitamin D supplements. In order to avoid vitamin D insufficiency, it is also necessary to maintain tight control over one's diabetic condition. We truly hope that this research will be a significant step forward in our knowledge of the relationship between vitamin D levels and type 2 diabetes mellitus, and that it will aid in the prevention of vitamin D insufficiency in diabetic patients.

\section{Reference}

1. Olokoba AB, Obateru OA, Olokoba LB. Type 2 Diabetes Mellitus: A Review of Current Trends. Oman Med J 2012;27(4):269-73.

2. Forouhi NG, Luan J, Cooper A et al. Baseline serum 25-hydroxy vitamin $\mathrm{D}$ is predictive of future glycaemic status and insulin resistance: the Medical Research Council Ely Prospective Study 1990 - 2000. Diabetes 2008;57:2619-25.

3. Chiu KC, Chu A, Go VL et al. Hypovitaminosis D is associated with insulin resistance and beta cell dysfunction. Am J Clin Nutr 2004;79:820-25.

4. Scragg $\mathrm{R}$, Sowers $\mathrm{M}$, Bell C. Serum 25hydroxyvitamin $\mathrm{D}$, diabetes, and ethnicity in the Third National Health and Nutrition Examination Survey. Third National Health and Nutrition Examination Survey. Diab Care 2004;27(12):2813-8.

5. Holick MF, Siris ES, Binkley $\mathrm{N}$ et al. Prevalence of vitamin D inadequacy among postmenopausal North American women receiving osteoporosis therapy. J Clin Endocrinol Metab 2005;90:3215-24.

6. Lips $\mathrm{P}$, Hosking D, Lippuner $\mathrm{K}$ et al. The prevalence of vitamin $\mathrm{D}$ inadequacy amongst women with osteoporosis: an international epidemiological investigation. J Intern Med 2006;260:245-54.

7. Pfotenhauer KM, Shubrook JH. Vitamin D deficiency, its role in health and disease, and current supplementation recommendations. J Am Osteopath Assoc 2017;117(5):301-5.

8. Laway BA, Kotwal SK, Shah ZA. Pattern of 25 hydroxy vitamin D status in North Indian people with newly detected type 2 diabetes: A prospective case control study. Indian J Endocrinol Meta 2014;18(5):726.

9. Alhumaidi M, Adnan AG, Dewish M. Vitamin D deficiency in patients with type-2 diabetes mellitus in southern region of Saudi Arabia. Maedica 2013;8(3):231.

10. Akshay Kumar SV, Nanda SK, Bharathy N, Ravichandran K, Dinakaran A, Ray L. Evaluation of vitamin $\mathrm{D}$ status and its correlation with glycated haemoglobin in type 2 diabetes mellitus. Biomedical Res, 2017, 28(1).

11. Kostoglou-Athanassiou I, Athanassiou P, Gkountouvas A, Kaldrymides P. Vitamin D and glycemic control in diabetes mellitus type 2. Therapeutic Adv Endocrinol Meta 2013;4(4):122-8.

12. Brijesh M, Saurav P. Prevalence of Vitamin D deficiency in type-2 Diabetes Mellitus patients and its correlation with glycaemic control. Int. J Bioas 2014;3:3313-7.

13. Modi KD, Ahmed MI, Chandwani R, Kumar KH. Prevalence of vitamin D deficiency across the spectrum of glucose intolerance. J Diabetes Meta Dis 2015;14(1):54.

14. Priya S, Singh A, Pradhan A, Himanshu D, Agarwal A, Mehrotra S. Association of Vitamin D and essential hypertension in a North Indian population cohort. Heart India 2017;5(1):7. 